Pacific Journal of Mathematics

INTEGRAL DOMAINS THAT ARE NOT EMBEDDABLE IN 


\title{
INTEGRAL DOMAINS THAT ARE NOT EMBEDDABLE IN DIVISION RINGS
}

\author{
JOHN DAUNS
}

A class of totally ordered rings $V$ is constructed having the property $1<\alpha \in V \Rightarrow 1 / \alpha \in V$, but such that $V$ cannot be embedded in any division ring.

1. Inverses in semigroup power series rings. This note has only one objective-to construct the above class of counterexamples (see [6]).

Notation 1.1. Throughout $\Gamma$ will be a totally ordered cancellative semigroup with identity $e ; R$ will denote any totally ordered division ring. If $\alpha: \Gamma \Rightarrow R$ is any function, then the support of $\alpha$ is the set $\operatorname{supp} \alpha=\{s \in \Gamma \mid \alpha(s) \neq 0\}$. The set $V=V(\Gamma, R)$ of all functions $\alpha$ such that $\operatorname{supp} \alpha$ satisfies the a.c.c. (ascending chain condition) form a totally ordered abelian group. If $\Gamma$ is cancellative, then under the usual power series multiplication (see [3]), $V$ is a totally ordered ring.

1.2. Any $1<\alpha \in V$ with $\alpha(s)=0$ for $s>e$ may be written as $\alpha=\alpha(e)(1-\lambda)$, where $1 \leqq \alpha(e)$ and $\lambda=\Sigma\{\lambda(\alpha) \alpha \mid a<e\}$. It will be shown that

$$
(1-\lambda)^{-1}=1+\lambda+\lambda^{2}+\cdots=1+\sum_{s} \Sigma^{\prime} \lambda(a(1) \lambda(a(2)) \cdots \lambda(a(n)),
$$

where the finite sum $\Sigma^{\prime}$ is over all integers and over all distinct $n$ tuples of $\Gamma^{n}$ satisfying $s=a(1) a(2) \cdots a(n)$ with each $a(i)<e$; the sum $\Sigma$ is over all $s<e$. To prove that $1 / \alpha \in V$ it suffices to establish conditions $(a)$ and $(b)$ below.

(a) For each $s \in \Gamma$, there are only a finite number of $n$ with $\lambda^{n}(s) \neq 0$;

(b) $\operatorname{supp}(1-\lambda)^{-1}$ satisfies the a.c.c.

Assuming (a) and (b), the main theorem follows at once. By adjoining an identity as in [8; p. 158] to the semigroup in [2] a semigroup that actually satisfies the hypothesis in (ii) below can be constructed.

MAIN THEOREM 1.3. If $\Gamma$ is a totally ordered cancellative semigroup with identity $e$ and $R$ any totally ordered division ring, then the power series ring $V=V(\Gamma, R)$ has the following properties:

(i) $1<\alpha \in V$ and $\alpha(s)=0$ for $s>e \Longrightarrow 1 / \alpha \in V$.

(ii) If in addition $\Gamma$ cannot be embedded in a group, then $V$ 
cannot be embedded in a division ring.

An already known result ([8; p. 135]) follows immediately from 1.3 (i).

COROLlaRY 1.4. If in addition $\Gamma$ is a group, then $V(\Gamma, R)$ is a division ring.

2. Proof of the main theorem. Assume 1.2 (a) or (b) fails. Then a lengthy but elementary argument shows there exists a doubly indexed matrix $\{a(i, j) \in \operatorname{supp} \lambda \mid 1 \leqq i<\infty ; 1 \leqq j \leqq n(i)\}$ such that the products $u(i)=a(i, 1) a(i, 2) \cdots a(i, n(i))$ of the rows form an infinite properly ascending chain. Eventually a contradiction will be derived from this. Without loss of generality assume $\Gamma \leqq e$.

DEFINITION 2.1. For any totally ordered semigroup $\Gamma$ with identity $e$ and any element $a \in \Gamma$ with $a \leqq e$, define a semigroup by

$$
\Gamma(a)=\left\{q \in \Gamma \mid \exists \text { an integer } m>0, q^{m} \leqq a\right\} .
$$

Lemma 2.2. With $\Gamma$ as above, for any $a(1), \cdots, a(m) \in \Gamma$ with each $a(j) \leqq e$, set $u=a(1) a(2) \cdots a(m)$ and define

$$
a^{*}=\min \{a(1), \cdots, a(m)\} \text {. }
$$

Then $\Gamma(u)=\Gamma\left(a^{*}\right)$.

2.3. Consider a fixed subset $L \subseteq \Gamma$ all of whose elements satisfy $L \leqq e$ and where $L$ satisfies the a.c.c., e.g., $L=\operatorname{supp} \lambda<e$. Consider an array of elements $A=\|a(i, j)\|$ with $\{a(i, j) \mid 1 \leqq i<\infty, 1 \leqq j \leqq$ $n(i)\} \subseteq L$, where repetitions in the $a(i, j)$ are allowed. Assume all $n(i) \geqq 2$. Define $u(i)=u(i, A)$ by

$$
u(i)=u(i, A)=a(i, 1) a(i, 2) \cdots a(i, n(i)) .
$$

Let $\mathscr{K}$ be the set of all such $A=\|a(i, j)\|$ for which $u(1)<u(2)<$ $\cdots<u(i)<\cdots$ is strictly ascending at each $i$. With each member $A=\|a(i, j)\| \in \mathscr{K}$, we next associate three objects

$$
\left\{a(i)^{*} \mid 1 \leqq i<\infty\right\}, m=m(A) \text {, and } \boldsymbol{G}=\boldsymbol{G}(A) \text {. }
$$

Define $a(i)^{*} \equiv \min \{a(i, j) \mid 1 \leqq j \leqq n(i)\}$. Note that $u(1)<u(2)<\cdots$ implies that $\Gamma\left(a(1)^{*}\right) \subseteq \Gamma\left(a(2)^{*}\right) \subseteq \Gamma\left(a(i)^{*}\right) \subseteq \ldots$. Thus since $L$ satisfies the a.c.c., there is a unique smallest integer $m \equiv m(A)$ such that the semigroups $G \equiv \Gamma\left(a(m)^{*}\right)=\Gamma\left(a(m+1)^{*}\right)=\cdots$ are all equal. The following schematic diagram of all these quantities may be helpful. 


$$
\begin{aligned}
& \Gamma\left(a(1)^{*}\right)=\Gamma(u(1)) \quad u(1)=a(1,1) a(1,2) \cdots a(1)^{*} \cdots a(1, n(1)) \\
& \text { กII } \\
& \Gamma\left(a(2)^{*}\right)=\Gamma(u(2)) \quad u(2)=a(2,1) a(2,2) \cdots a(2)^{*} \cdots a(2, n(2)) \\
& \Gamma\left(a(m)^{*}\right)=\Gamma(u(m)) \quad u(m)=a(m, 1) a(m, 2) \cdots a(m)^{*} \cdots a(m, n(m)) \\
& \text { II } \\
& \boldsymbol{G}=\Gamma(u(m+1)) \text {. }
\end{aligned}
$$

2.4. Among the elements of $\mathscr{K}$, let $\mathscr{N} \subset \mathscr{K}$ be all those $A=$ $\|a(i, j)\|$ such that this associated $\boldsymbol{G}=\boldsymbol{G}(A)$ is as big as possible and call this particular $\boldsymbol{G} \equiv \boldsymbol{M}$. If $\mathscr{K} \neq \varnothing$, also $\mathscr{N} \neq \varnothing$. Define $\bar{a}=$ $\max \left\{a(m)^{*} \mid A \in \mathscr{K}, m=m(A)\right\}$. Pick and element $B=\|b(i, j)\| \in \mathscr{N}$. Then by our choice of $\boldsymbol{M}, \Gamma(\bar{a})=\boldsymbol{M}$. Thus $\boldsymbol{M}=\boldsymbol{G}(B)=\Gamma\left(b(i)^{*}\right)=$ $\Gamma(b(i, j))=\Gamma(u(i))=\Gamma(\bar{a})$ for $i \geqq m(B) \equiv m$. Finally, with each element $B$ of $\mathscr{N}$, we associate an integer $r=r(B)$. Since $\bar{a} \in \Gamma(u(m))$, there is a unique smallest integer $r \equiv r(B) \geqq 1$ such that $\bar{a}^{r} \leqq u(m)<\bar{a}^{r-1}$.

2.5. By omitting some of the rows of $B$ and renumbering the remaining ones, it may be assumed as a consequence of the a.c.c. without loss of generality that $m=1$, and also that $b(1)^{*} \geqq b(2)^{*} \geqq \cdots$ is not ascending. Each $u(i)$ is of one of the following three forms:

$$
\begin{aligned}
& u(i)=q(i) b(i)^{*}, \\
& u(i)=b(i)^{*} w(i), \\
& u(i)=q(i) b(i)^{*} w(i),
\end{aligned}
$$

where the $q(i), w(i)$ are certain products of the $b(i, j)$. If there are an infinite number of $u(i)$ of the forms (1) or (2), then since

$$
\begin{aligned}
u(i+1) & =q(i+1) b(i+1)^{*}>u(i)=q(i) b(i)^{*}, b(i+1)^{*} \leqq b(i)^{*} \\
& =q(i+1)>q(i) ;
\end{aligned}
$$

it follows (after omitting some rows and renumbering) that there is a properly infinite ascending chain:

Case 1. $q(1)<q(2)<\cdots$;

Case 2. $w(1)<w(2)<\cdots$.

If neither Case 1 nor Case 2 applies, then

$$
\begin{gathered}
u(i+1)=q(i+1) b(i+1)^{*} w(i+1)>q(i) b(i)^{*} w(i) \\
\text { and } b(i+1)^{*} \leqq b(i)^{*}
\end{gathered}
$$

implies that one of the inequalities $q(i+1)>q(i)$ or $w(i+1)>w(i)$ 
must necessarily hold. It is asserted that there is a subsequence $\{i(k) \mid k=1,2, \cdots\}$ such that

Case 3. either (a): $q(i(1))<q(i(2))<\cdots$

$$
\text { or }(\mathrm{b}): \quad w(i(1))<w(i(2))<\cdots \text {. }
$$

For if not, then the a.c.c. must hold in both the sets $\{q(i)\}$ and $\{w(i)\}$. Then by omitting some rows and renumbering the remaining ones it may be assumed that we have an element $B$ in $\mathscr{N}$ with $q(1) \geqq q(2) \geqq \cdots$ and $w(1) \geqq w(2) \geqq \cdots$. However, then

$$
q(1) b(1)^{*} w(1) \geqq q(2) b(2)^{*} w(2) \geqq \cdots
$$

gives a contradiction.

2.6. We may assume $q(1)<q(2)<\cdots$ or $w(1)<w(2) \cdots$ are properly ascending, depending on which of the Cases 1, 2,3(a) or 3(b) is applicable. Set $t=r(B)$, so that $\bar{a}^{t} \leqq u(m)=u(1) \leqq u(i)$.

2.7. It is next shown that either $q(i) \geqq \bar{a}^{t-1}$ or $w(i) \geqq \bar{a}^{t-1}$ holds for all $i$. Suppose that the following holds.

Case 1. $q(1) b(1)^{*}<q(2) b(2)^{*}<\cdots$;

$$
\begin{aligned}
q(1) & <q(2) \quad<\cdots ; \\
b(1)^{*} & \geqq b(2)^{*} \geqq \cdots \cdot
\end{aligned}
$$

Then $\bar{a}^{t} \leqq u(1) \leqq u(i)=q(i) b(i)^{*}$, and $\bar{a} \geqq b(i)^{*}$ implies that

$$
\bar{a}^{t-1} \leqq q(1) \leqq q(i) \text {. }
$$

(For if $\bar{a}^{t-1}>q(i)$, then $\bar{a} \geqq b(i)^{*}$ implies that $\bar{a}^{t}>q(i) b(i)^{*}$.) (If $t=$ 1 , then $\bar{a}^{0}=e$.) Similarly, in Case 2 also $\bar{a}^{t-1} \leqq w(1) \leqq w(i)$.

Only Case 3(b) will be proved, since 3(a) is entirely parallel.

Case $3(\mathrm{~b}) . \quad q(1) b(1)^{*} w(1)<q(2) b(2)^{*} w(2)<\cdots$;

$$
\begin{aligned}
w(1) & <\quad w(2)<\cdots ; \\
b(1)^{*} & \geqq b(2)^{*} \geqq \cdots .
\end{aligned}
$$

Then again $\bar{a}^{t} \leqq u(1) \leqq u(i)=q(i) b(i)^{*} w(i)$ and $\bar{a} \geqq b(i)^{*} \geqq q(i) b(i)^{*}$ imply that $\bar{a}^{t-1} \leqq w(1) \leqq w(i)$. (Otherwise, if $\bar{a}^{t-1}>w(i)$, then $\bar{a}^{t}>$ $q(i) b(i)^{*} w(i)$.)

The basic idea motivating the proof is that for $B \in \mathscr{N}$, a new $C \in \mathscr{N}$ can be constructed with $r(C) \leqq r(B)-1$. 
2.8. Thus either $q(1)<q(2)<\cdots$ and all $q(i) \geqq \bar{a}^{t-1}$; or $w(1)<$ $w(2)<\cdots$ and all $w(i) \geqq \bar{a}^{t-1}$. Assume the latter. Let

$$
C=\|c(i, j)\| \in \mathscr{K}
$$

be defined by taking as its $i$-th row all the $b(i, j)$ appearing in $w(i)$. (In view of $w(1)<w(2)<\cdots$, there does not exist an infinite number of rows of $C$ containing only one element. By omitting a finite number of rows it may be assumed that all rows of $C$ contain two or more elements of $L$.) Define $c(i)^{*} \equiv \inf \{c(i, j) \mid j \geqq 1\}$. Since $b(i)^{*} \leqq c(i)^{*} \leqq \bar{a}$, it follows that

$$
\boldsymbol{M}=\Gamma\left(b(i)^{*}\right) \subseteq \Gamma\left(c(i)^{*} \subseteq \Gamma(\bar{a})=M .\right.
$$

Consequently, $\boldsymbol{G}(C)=\boldsymbol{M}$ and $C \in \mathscr{N}$. Since $w(1) \geqq \bar{a}^{t-1}, r(C) \leqq t-1$. By repetition of this process, we may reduce the $r$ to one so that finally $\bar{a}^{r}=\bar{a} \leqq w(1)<w(2) \cdots$. Since all $c(i, j) \in L$ satisfy $c(i, j) \leqq e$ and since $w(i)$ is a product of these, it follows that $\bar{a} \geqq c(i)^{*} \geqq w(i)$. Thus $\bar{a}=w(1)=w(2)=\cdots$ gives a contradiction. Thus $\mathscr{K}=\varnothing$ and the main theorem has been proved.

\section{REFERENCES}

1. A. J. Bowtell, On a question of Mal'cev, J. of Algebra 7 (1967), 126-139.

2. C. G. Chehata, On an ordered semigroup, J. London Math. Soc. 28 (1953), 353-356.

3. P. Conrad and J. Dauns, An embedding theorem for lattice ordered fields, Pacific

J. Math. 30 (1969), 385-398.

4. J. Dauns, Representation of f-rings, Bull. Amer. Math. Soc. 74 (1968), 249-252.

$5 . \quad$, Representation of l-groups and f-rings, Pacific J. Math. 31 (1969), 629-654.

6 . - Semigroup power series rings (to appear).

7. Embeddings in division rings (to appear).

8. L. Fuchs, Partially ordered algebraic systems, Pergammon Press, 1963.

9. R. E. Johnson, Extended Mal'cev domains, Proc. Amer. Math. Soc. 21 (1969), 211213.

10. A. A. Klein, Rings nonembeddable in fields with multiplicative semigroups embeddable in groups, J. of Algebra 7 (1967), 100-125.

11. B. H. Neumann, On ordered division rings, Trans. Amer. Math. Soc. 66 (1949), 202-252.

Received September 3, 1969, and in revised form December 13, 1969.

TUlane University

New Orleans, Louisiana 



\section{PACIFIC JOURNAL OF MATHEMATICS}

EDITORS

H. SAMELSON

Stanford University

Stanford, California 94305

RichaRd PIERCE

University of Washington

Seattle, Washington 98105
J. DUGUNDJI

Department of Mathematics

University of Southern California

Los Angeles, California 90007

RICHARD ARENS

University of California

Los Angeles, California 90024

\section{ASSOCIATE EDITORS}

E. F. BECKENBACH

B. H. NeumanN

F. WOLF

K. YOSHIDA

\section{SUPPORTING INSTITUTIONS}

UNIVERSITY OF BRITISH COLUMBIA

CALIFORNIA INSTITUTE OF TECHNOLOGY

UNIVERSITY OF CALIFORNIA

MONTANA STATE UNIVERSITY

UNIVERSITY OF NEVADA

NEW MEXICO STATE UNIVERSITY

OREGON STATE UNIVERSITY

UNIVERSITY OF OREGON

OSAKA UNIVERSITY

UNIVERSITY OF SOUTHERN CALIFORNIA
STANFORD UNIVERSITY

UNIVERSITY OF TOKYO

UNIVERSITY OF UTAH

WASHINGTON STATE UNIVERSITY

UNIVERSITY OF WASHINGTON

AMERICAN MATHEMATICAL SOCIETY CHEVRON RESEARCH CORPORATION TRW SYSTEMS

NAVAL WEAPONS CENTER 


\section{Pacific Journal of Mathematics}

May, 1970

Johan Aarnes, Edward George Effros and Ole A. Nielsen, Locally compact

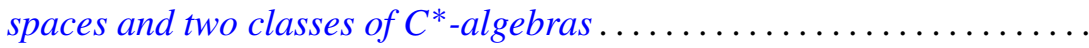

Allan C. Cochran, R. Keown and C. R. Williams, On a class of topological

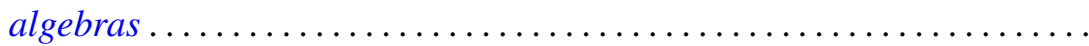

John Dauns, Integral domains that are not embeddable in division rings ....

Robert Jay Daverman, On the number of nonpiercing points in certain

crumpled cubes.....................................

Bryce L. Elkins, Characterization of separable ideals ................

Zbigniew Fiedorowicz, A comparison of two naturally arising uniformities

on a class of pseudo-PM spaces ...........................

Henry Charles Finlayson, Approximation of Wiener integrals of functionals

continuous in the uniform topology ........................

Theodore William Gamelin, Localization of the corona problem ...........

Alfred Gray and Paul Stephen Green, Sphere transitive structures and the

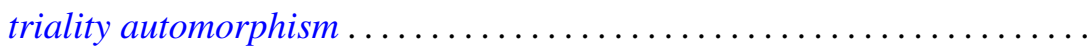

Charles Lemuel Hagopian, On generalized forms of aposyndesis ..........

J. Jakubík, On subgroups of a pseudo lattice ordered group ...............

Cornelius W. Onneweer, On uniform convergence for Walsh-Fourier

series..................................

Stanley Joel Osher, On certain Toeplitz operators in two variables ...

Washek (Vaclav) Frantisek Pfeffer and John Benson Wilbur, On the

measurability of Perron integrable functions............

Frank J. Polansky, On the conformal mapping of variable regions...

Kouei Sekigawa and Shûkichi Tanno, Sufficient conditions for a Riemannian manifold to be locally symmetric ...................

James Wilson Stepp, Locally compact Clifford semigroups ....

Ernest Lester Stitzinger, Frattini subalgebras of a class of solvable Lie

algebras ................................

George Szeto, The group character and split group algebras...

Mark Lawrence Teply, Homological dimension and splitting torsion

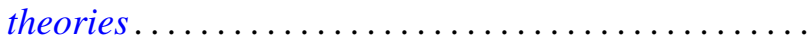

David Bertram Wales, Finite linear groups of degree seven. II ...

Robert Breckenridge Warfield, Jr., An isomorphic refinement theorem for

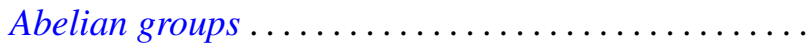

James Edward West, The ambient homeomorphy of an incomplete subspace

of infinite-dimensional Hilbert spaces................

Peter Wilker, Adjoint product and hom functors in general topology ...

Daniel Eliot Wulbert, A note on the characterization of conditional 